\title{
Une anomalie du gène codant pour le ligand du CD40 est responsable du déficit immunitaire caractérisé par une hyper-IGM liée à I'X chez I'homme
}

Un article de synthèse paru dans médecine/sciences en juillet 1992 [1] rappelait combien la caractérisation des gènes responsables des déficits immunitaires de transmission liée au chromosome $\mathrm{X}$ promettait d'apporter de nouveaux enscignements sur les mécanismes qui gouvernent la régulation de notre systc̀me immunitaire. Coup sur coup, deux de ces gènes viennent d'être identifiés. Le premier [2], dont l'anomalie est responsable de l'agammaglobulinémie de Bruton, caractérisée par un blocage de différenciation lymphocytaire B au stade pré-B, code pour une protéine tyrosine-kinase spécifiquement exprimée dans les lymphocytes B et les cellules myélomonocytaires (m/s $n^{\circ} 3$, vol. 9, p. 332). Découvertes qui, comme d'habitude, suscitent de multiples questions : comment et à quel stade de l'ontogénie B cette protéine agit-elle ? avec quels cofacteurs et substrats ? quelle est la cible de son action ? - autant d'axes de recherche qui permettront d'accrôitre encore notre connaissance sur les phénomènes intervenant dans la différenciation lymphocytaire.

\section{Le syndrome d'hyper-IgM}

Lc second gc̀ne identifié est responsable du syndrome d'hyper-IgM, hypo$\operatorname{Ig} \mathrm{A}, \operatorname{IgG}, \operatorname{IgE}$ ou dysgammaglobulinémie de type I [3-6]. Ce déficit immunitaire, comme son nom l'indique, se caractérise principalement par un défaut apparent de la commutation isotypique ou switch. Les enfants atteints de ce déficit ont un taux normal ou le plus souvent élevé des Ig de types $\mathrm{M}$ et $\mathrm{D}$ en réponse aux stimulations antigéniques, alors que les swilch pour être produites sont absentes. Cliniquement [7], ces enfants se présentent avec des infections à répétition, souvent dès la première année de vie, qui touchent les bronches, la sphère ORL, la peau, l'intestin. Les lymphocytes $T$ et $B$ circulants sont en nombre normal, bien qu'il soit fréquent de trouver une hyperplasie des ganglions lymphatiques avec une absence des centres germinatifs B. On sait qu'il existe des formes autosomiques et des formes liées à l'X de cc déficit immunitaire.

Bien que la cellule effectrice du switch soit le lymphocyte $\mathrm{B}$, une anomalie du lymphocyte $\mathrm{T}$ intervenant dans la coopćration T/B lymphocytairc était soupçonnée. Trois arguments plaidaient pour cette hypothèse :

- les patients atteints du syndrome d'hyper-IgM sont sensibles à des agents infectieux bien particuliers (Pneumocystis carinii, cryptosporidie...) que l'on retrouve au cours des déficits immunitaires plutôt de type cellulaire (des lymphocytes $\mathrm{T}$ ) qu'humoral (des lymphocytes B);

- les femmes, conductrices obligatoires de ce syndrome, prćsentent dans leurs lymphocytes B comme dans les autres cellules de l'organisme, un profil d'inactivation au hasard de leurs chromosomes X rendant peu probable une anomalie intrinsèque des lymphocytes $B$; - enfin, Mayer et al., en 1986 [8], avaient montré que les lymphocytes $B$ des patients atteints du syndrome d'hyper-IgM, cultivés en présence d'une lignée $T$ lymphomateuse d'un syndrome de Sézary, étaient capables de se différencier en cellules sécrétant les différents isotypes d'Ig ( $\operatorname{IgG}, \mathrm{A}$ et $\mathrm{E})$, ce qui tendait également à inno- center une anomalie princeps du lymphocyte B.

\section{La molécule CD40}

La nécessité d'une coopération entre les lymphocytes $\mathrm{T}$ et les lymphocytes $\mathrm{B}$, à l'origine de nombreuses fonctions $\mathrm{B}$, est connue depuis longtemps. Elle peut être effectuée soit par des facteurs solubles, les cytokines, soit nécessiter un contact cellulaire entre ces deux types de lymphocytes. C'est le cas de la coopération $T / B$ qui utilise la voie du CD40 [9]. L'activation de la molécule CD40 exprimée très tôt à la surface du lymphocyte $\mathrm{B}$ permet à la fois la croissance à long terme de ces cellules et, en association avec des cytokines, leur différenciation vers les cellules sécrétrices d'Ig des différentes classes. Cette molécule appartient à une famille de récepteurs de facteurs de croissance [10] avec, en particulier, les récepteurs aux tumor necrosis factor (TNF $\alpha$ et $\beta$ ), et le récepteur de basse affinité à un facteur de croissance du système nerveux (NGFR). Les membres de cette famille ont une caractéristique originale, celle de pouvoir s'associer à plusieurs ligands. C'est le cas des récepteurs du TNF qui peuvent $s$ 'associer indifféremment avec le $\mathrm{TNF} \alpha$ ou $\beta$ ou du récepteur à faible affinité du NGF qui lie à la fois le NGF et deux autres facteurs neurotropes le brain-derived neurotrophic factor (BDNF) et la neutrophine-3 (NT-3).

De plus, les ligands déjà identifiés des récepteurs appartenant à cette famille sont actifs sous forme di- ou trimérique.

\section{Le ligand de la molécule CD40}

Très récemment, le ligand de la molécule CD40 a été identifié chez la sou- 


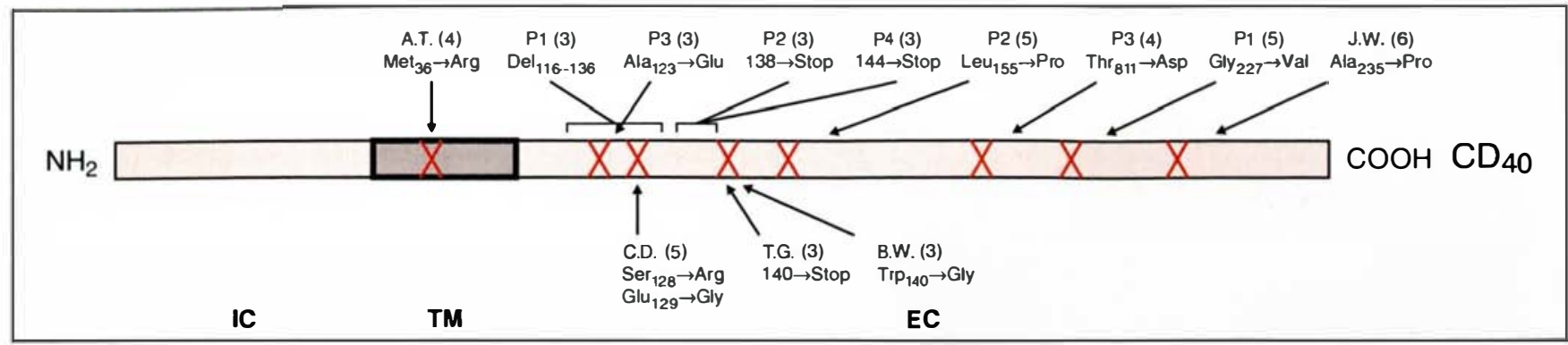

Figure 1. Représentation schématique de la région codante du ligand de CD40 et des différentes mutations ponctuelles $(X)$ ou délétions $(M)$ retrouvées chez 12 patients étudiés [3-6] présentant un syndrome d'hyper-IgM. IC : domaine intracellulaire; TM : région transmembranaire; EC : domaine extracellulaire.

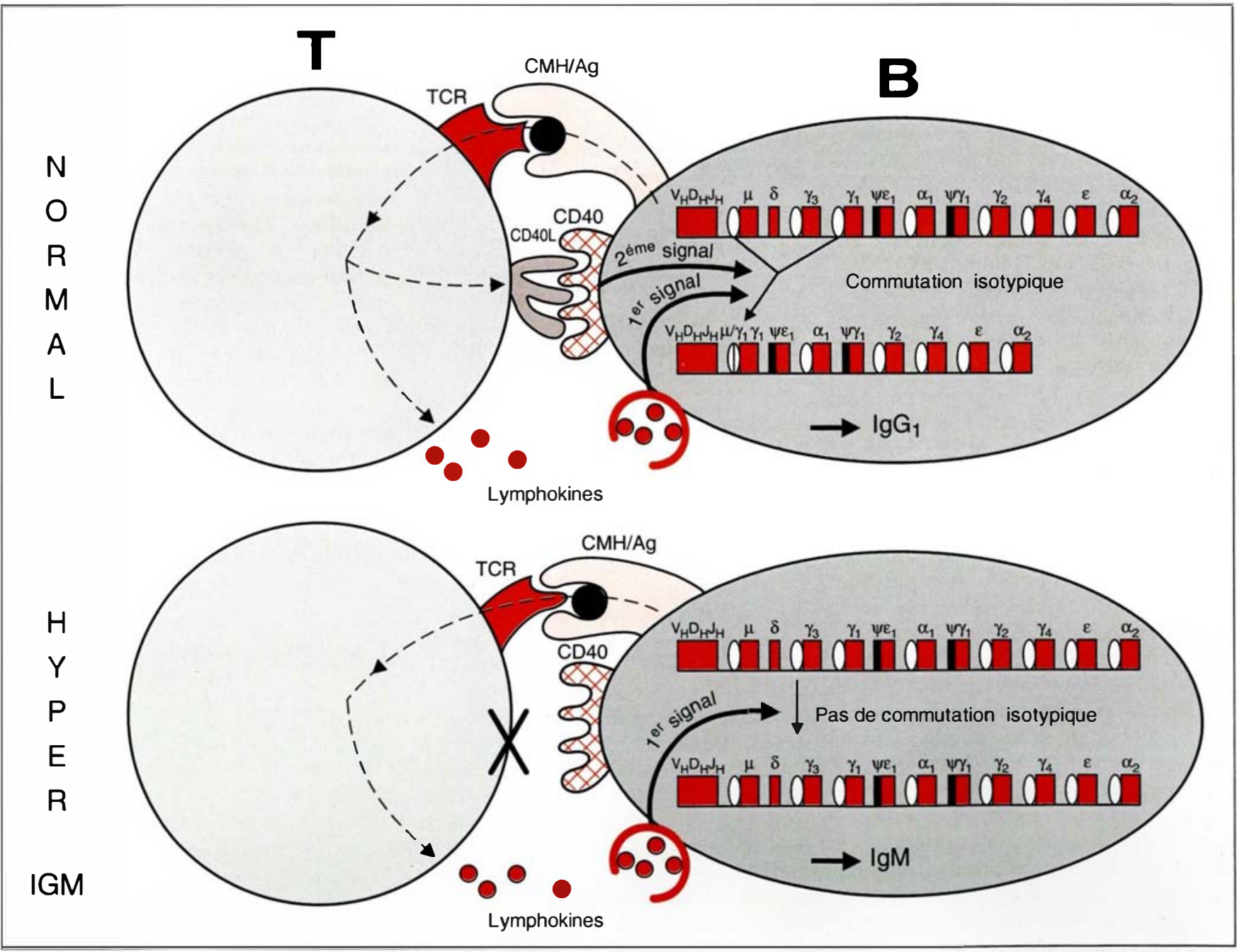

Figure 2. Le lymphocyte $T$ activé par le complexe antigène/complexe majeur d'histocompatibilité présenté par le macrophage ou le lymphocyte B exprime à sa surface le ligand de CD40 et sécrète des lymphokines. La commutation isotypique des différents chaînes constantes des immunoglobines rectangles rouges, par l'intermédiaire de leur région de switch ovales blancs, nécessite un double signal relayé d'une part, par la molécule CD40, d'autre part, par des lymphokines. La spécificité de la lymphokine détermine l'isotype produit IIIA, pour IgG dans l'exemple représenté et pour IgE, TGF $\beta$ pour IgA). En l'absence d'un des deux signaux (CD4O L dans le syndrome hyper IgM lié à l'X), la commutation isotypique n'a pas lieu et seules des IgM sont produites. CD4OL : CD40-ligand.

$m / s n^{\circ} 4$ vol. 9, avril 93 
ris [11] puis, quelques mois après, chez l'homme [12] par plusieurs équipes. Il s'agit d'une protéine de $35 \mathrm{kDa}$ exprimée à la surface des lymphocytes $\mathrm{T}$ activés, surtout de type auxiliaire helper $\left(\mathrm{CD}^{+}\right)$, et sur une partie des lymphocytes $\mathrm{T}$ cytotoxiques ou suppresseurs $\left(\mathrm{CD} 8^{+}\right)$.

Cette protéine partage de nombreux points communs avec les $\mathrm{TNF} \alpha$ et $\beta$ (structure, association en trimère), ce qui laisse penser que les ligands de ces facteurs de croissance pourraient également eux-mêmes appartenir à une même famille de gènes.

\section{Anomalie du ligand de CD40 chez les patients atteints d'hyper-IgM liée à l'X}

La localisation sur le bras long du chromosome $X$ en q26-q27 du gène codant pour le CD40L fut certainement le point décisif qui permit d'envisager qu'une anomalie de ce gène pouvait être responsable du syndrome d'hyper-IgM de transmission liée au chromosome $\mathrm{X}$ chez l'homme. Ce déficit immunitaire a en effet été localisć précédemment dans cette même région par étude de liaison dans les familles à risque à l'aide de marqueurs polymorphiques de l'X. Quatre groupes s'attachèrent à rechercher et viennent de publier simultanément des anomalies du ligand de CD40 chez les patients présentant un syndrome d'hyper-IgM [3-6]. Les lymphocytes T de tous les patients étudiés montrent une incapacité complète (ou partielle dans un cas) à fixer une forme soluble de la molécule CD40. En revanche, la stimulation de leurs lymphocytes B par la voie du CD40 à l'aide d'anticorps spécifiques permet de rétablir la prolifération des lymphocytes B de ces patients ainsi que leur différenciation en cellules sécrétant les différentes classes d'immunoglobulines en présence des cytokines appropriées. Les transcrits du ligand de CD40 de 13 patients ont été séquencés et 12 sujets présentent des anomalies, toutes différentes, sous forme de mutations ponctuelles (neuf cas) ou de délétions (trois cas) (figure 1).

Ces anomalies sont bien en relation avec l'absence de coopération T/B utilisant la voie du CD40. Des lignées cellulaires de rein transfectées avec des
ADN clonés codant pour les formes mutantes du ligand de CD40 sont incapables de lier la molécule CD40 et d'induire un switch de cellules B normales alors que la transfection de ces mêmes lignées par l'ADN normal du ligand de CD40 le fait. Chez les mères de trois des quatre sujets dans notre étude [3], le caractère héréditaire est démontré par la détection de la même anomalic chez ces femmes que chez leur fils. Le quatrième cas semble être une anomalie apparue de novo chez le patient. Chez les femmes conductrices de ce déficit, une double population lymphocytaire $T$ semble exister. En effet, l'identification d'un microsatellite très polymorphique dans la région 3' non traduite du ligand de CD40 a permis de montrer [3] que, chez une femme conductrice informative pour ce marqueur, existaient une population $\mathrm{T}$ négative, ne fixant pas à sa surface la molécule CD40 soluble et une population $\mathrm{T}$ positive, fixant la molécule CD40. La population négative transcrit l'allèle muté du chromosome $\mathrm{X}$ de cette femme, alors que la population positive transcrit l'allèle sain. $\mathrm{Ce}$ microsatellite intragénique s'avère de plus extrêmement précieux dans le cadre du diagnostic, diagnostic anténatal et de conductrice, aucun autre moyen n'étant disponible en dehors de l'identification dans chaque cas de l'anomalie génique propre.

\section{Quelles autres fonctions pour le cou-} ple CD40-CD40L

Il semble maintenant clair qu'une anomalic du gène codant pour le ligand de la molécule CD40 puisse être responsable du syndrome d'hyper-IgM lié à l'X chez l'homme, et que l'interaction du CD40 avec son ligand soit un maillon essenticl de la différenciation $B$ au niveau de la commutation isotypique. Un certain nombre de questions restent cependant non résolues. Les patients présentant un syndrome hyper-IgM associent souvent une neutropénie, des phénomènes d'autoimmunité et des lymphomes de type $\mathrm{T}$ ou B [7]. Il est possible que le couple CD40-ligand de CD40 ait un rôle plus large dans le développement du système immunitaire que la seule coopération $\mathrm{T} / \mathrm{B}$ en vue de la commutation isotypique. Cela apparaît d'autant plus vraisemblable que la molécule CD40 est exprimée non seulement sur les lymphocytes $\mathrm{B}$, mais également sur nombre d'épithélium, et sur les cellules de la lignée histiocytaire. De même, le ligand de CD40 pourrait être retrouvé sur d'autres types cellulaires. Pourquoi ne pas imaginer qu'en l'absence de son propre ligand, la molécule CD40 puisse également interagir avec d'autres ligands de la même famille comme le TNF, dont on connaît le rôle important dans les phénomènes d'auto-immunité et de tumorigenèse.

\section{Perspectives thérapeutiques}

Quels peuvent être dans le futur les progrès thérapeutiques que l'on peut attendre de l'identification de ce gène ? A l'heure actuelle, le traitement du syndrome d'hyper-IgM consiste en l'injection répétée de gammaglobulines et en une antibiothérapie adaptée. L'injection sous forme soluble du ligand de CD40 pourrait permettre aux patients de sécréter leurs propres immunoglobulines spécifiques. Le risque de dévclopper une réaction anticorps contre cette molécule n'est cependant pas à négliger. C'est probablement par le biais de modèles animaux du syndrome d'hyper-IgM que pourront être expérimentées les approches thérapeutiques utilisant les protéines ou les gènes recombinants

\section{James P. Di Santo Geneviève de Saint-Basile} Inserm U. 132, hôpital des Enfants-Malades, 149, rue de Sèvres, 75743 Paris Cedex 15, France.

\section{TIRÉS A PART}

G. de Saint-Basile 


\section{RÉFÉRENCES}

1. De Saint Basile G, Fisher A. Un modèle d'ćtude de la différenciation lymphocytaire : les déficits immunitaires héréditaires lićs au chromosome X. médecine/sciences $1992 ; 8$ : 562-71.

2. Vetrie D, Vorechovskyt I, Sideras P, Holland J, Davies A, Flinter F, Hammarström L, Kinnon C, Levinsky R, Bobrow M, Smith CIE, Bentley DR. The gene involved in Xlinked agammaglobulinaemia is a member of the src family of protein-tyrosine kinases. Nature 1993 ; 361 : 226-33.

3. Di Santo JP, Bonnefoy JY, Gauchat JF, Fischer A, de Saint-Basile G. CD40 ligand mutations in X-linked immunodeficiency with hyper-Ig M. Nature 1993 ; 361 : 541-3

4. Korthauer U, Graf D, Mages HW, Brièrc F, Padayachec M, Malcolm S, Ugazio AG, Notarangelo LD, Levinsky RJ, Kroczek RA. Defective expression of T-cell CD40 ligand causes X-linked immunodeficiency with hyperIgM. Nature $1993 ; 361$ : 539-41.

5. Allen RC, Armitage RJ, Conley ME, Rosenblatt H, Jenkins NA, Copeland NG, Bedell MA, Edelhoff S, Disteche CM, Simoneaux DK, Fanslow WC, Belmont J, Spriggs MK. CD40 ligand gene defects responsible for X-linked hyper-IgM syndrome. Science 1993 ; 259 : 990-3.

6. Aruffo A, Farrington M, Hollenbaugh D, Li X, Milatovich A, Nonoyama S, Bajorath J, Grosmairc LS, Stenkamp R, Neubaucr M, Roberts RL, Noclle RJ, Ledbetter JA, Francke U, Ochs HD. The CD40 ligand, gp39, is defective in activated $\mathrm{T}$ cells from patients with $\mathrm{X}$-linked hypcr-IgM syndrome. Cell 1993 ; 72 : 291-300.

7. Notarangelo LD, Duse M, Ugazio AG. Immunodeficiency with hyper-IgM (HIM). Immunodef Rev 1992; 3 : 101-22.

8. Mayer L, Kwan SP, Thompson C, Ko HS, Chiorazzi N, Waldmann T, Rosen F. Evidence for a defect in "switch" $T$ cells in patients with immunodeficiency and hyperimmunoglobulinemia M. N Engl J Med 1986 ; 314 : 409-13.

9. Noelle RJ, Ledbetter JA, Aruffo A. CD40 and its ligand, an essential ligand-receptor pair for thymus-dependent B-cell activation. Immunol Today 1992; 13 : 431-3.

10. Mallett S, Barclay AN. A new superfamily of cell surface proteins related to the nerve growth factor receptor. Immunol Today $1991 ; 12: 220-3$.

11. Armitage RJ, Fanslow WC, Strockbine L, Sato TA, Clifford KN, Macduff BM, Anderson DM, Gimpel SD, Davis-Smith T, Maliszewski CR, Clark EA, Smith CA, Grabstein KH, Cosman D, Spriggs MK. Molecular and biological characterization of a murine ligand for CD40. Nature $1992 ; 357: 80-2$.

12. Gauchat JF, Aubry JP, Mazzei G, Life $P$, Jomote $T$, Elson G, Bonnef oy JY. Human CD40-ligand : molecular cloning, cellular distribution and regulation of expression by factors controlling IgE production. FEBS Lett 1993 ; 315 : 259-66. 